\title{
Primary Malignant Lymphoma of the Uterus: A Case Report and Review of the Literature
}

\author{
Margriet Samama ${ }^{a} \quad$ Mariette van Poelgeest $^{b}$ \\ ${ }^{a}$ Department of Gynaecology, Sint Franciscus Gasthuis, Rotterdam, and \\ ${ }^{b}$ Department of Gynaecology, Leiden University Medical Center, Leiden, \\ The Netherlands
}

\section{Key Words}

Primary malignant lymphoma · Uterus · Diffuse large B-cell lymphoma

\begin{abstract}
Primary malignant lymphomas in the female genital tract are rare. Most cases are nonHodgkin lymphomas of which diffuse large B-cell lymphomas are most commonly seen. Symptoms are associated with other, more common diseases; therefore, a doctors' delay can be expected. In this case a woman presented with complaints of urinary obstruction due to a large tumour in the pelvic area. A laparotomy was performed. A very large tumour of the uterus was found with adherence to the pelvic wall and urinary bladder. Diagnostic histological examination showed a diffuse large B-cell lymphoma. Treatment with R-CHOP chemotherapy was started shortly after the operation. The treatment of patients with a primary malignant lymphoma of the uterus should be individualized with the following options: surgery, radiotherapy and/or chemotherapy.
\end{abstract}

\section{Introduction}

Malignant lymphomas are occasionally found in the uterus or cervix; however, primary malignant lymphomas in the female genital tract are rare [1]. The most common diagnosis is diffuse large B-cell lymphoma [1,2]. Main symptoms are abnormal vaginal blood loss, abdominal pain and/or urinary obstruction. Most of these reported symptoms are associated with more common diseases in gynaecology; as a result it takes longer before the correct diagnosis can be made.

There are few articles available on the optimal approach of this uncommon disease [3]. Here, a case report and review of literature are presented for the diagnostic and therapeutic options for a patient with a primary malignant lymphoma of the uterus. 


\section{Case Report}

A 79-year-old woman presented with urinary obstruction in another hospital. Physical examination revealed a large pelvic tumour reaching up to the umbilical level. A blind cervical smear was taken, showing slight atypical cells. Ultrasonography showed a large pelvic tumour, no distinction could be made from uterus, bladder or ovaries. Laboratory data showed an elevated ESR $61 \mathrm{~mm} / \mathrm{h}$, urea $203 \mathrm{mg} / \mathrm{dl}$, creatinine $5.3 \mathrm{mg} / \mathrm{dl}$, potassium $21.89 \mathrm{mg} / \mathrm{dl}$, and an abnormal haemoglobin level 9.3 $\mathrm{g} / \mathrm{dl}$. Tumour markers (CA-125, AFP, CEA, and B-HCG) were all within the normal range. Magnetic resonance imaging (MRI) scan of the abdomen revealed a large pelvic mass, most likely the uterus, which compressed the left and right ureter, and bilateral pelvic lymphadenopathy. A cystoscopy was performed, showing no signs of tumour invasion in the bladder, and no other abnormalities. An attempt to insert double J ureteral stents was unsuccessful. Therefore a bilateral percutaneous nephropyelostomy was performed. The patient received potassium supplementation once and two packed cells, after which the patient's clinical condition improved.

The patient was referred to our hospital. The differential diagnosis included a uterine sarcoma or carcinosarcoma. A diagnostic laparotomy was performed. There was a very large tumour from the uterus with adherence to the urinary bladder and both pelvic walls. An incisional biopsy was taken from the anterior wall of the uterus, which caused a blood loss of 1,000 ml. It was decided to wait for the pathological-anatomical results and the abdomen was closed.

Histological examination showed a diffuse large B-cell lymphoma from the uterus (fig. 1).

Some days after the operation a tumour was noticed in the patient's neck, with a diameter of $5 \mathrm{~cm}$. A biopsy was performed of this tumour, which showed a localization of the B-cell lymphoma. A bone marrow biopsy was taken, which showed normocellular bone marrow.

A computer tomography (CT) scan of the thorax and abdomen showed an inhomogeneous mass in the left hemisphere of the thyroid with retrosternal expansion, multiple pulmonary embolies, and besides the known tumour mass of the uterus, a hypodense lesion in the spleen.

Treatment was started with series of rituximab, cyclophosphamide, adriamycin, vincristine and prednisone (R-CHOP) chemotherapy under supervision of the haematology department. After 3 courses of chemotherapy a clear regression was seen of all tumour localizations and the nephrostomy catheters were removed. After 6 courses a control CT scan was performed, which showed regression of all intra-abdominal lymphoma localizations; however, the thyroidal localization remained somewhat enlarged (fig. 2). A fine needle biopsy was taken, showing normal thyroid tissue, no localization of lymphoma cells. Unfortunately, the patient died of cardiac arrest 9 months after treatment.

\section{Discussion}

Primary malignant lymphomas of the uterus or cervix are rare. In most cases of lymphomas of the uterus it concerns secondary involvement of the disease [3]. Less than $1 \%$ of the primary malignant lymphomas occur in the female genitalia [2]. Fox and More [4] defined three criteria for primary uterine lymphoma, which are: (1) clinically confined to the uterus, (2) no evidence of leukaemia, and (3) a fairly long interval between the primary uterine lymphoma and the secondary tumour. Most cases of primary uterine lymphoma concern non-Hodgkin lymphoma, usually diffuse large Bcell lymphoma. Immunohistochemical analysis distinguishes B- from T-cell lymphoma. In case of the diagnosis lymphoma, bone marrow biopsy must follow to exclude leukaemia $[1,5]$.

Most common symptoms of primary uterine lymphoma are abnormal vaginal blood loss or discharge $[3,5]$ as well as abdominal pain or mass effect as reported in this case. PAP smears are usually not abnormal, because primary lymphoma of the cervix or 
uterus arises in the stroma, and not in the epithelium. Presenting symptoms are not characteristic for uterine lymphoma, as they occur in many more diseases; therefore, it can take longer to make the right diagnosis $[3,4]$.

In case of radiological examination, MRI is recommended before CT scan as it is more discerning in differentiating cervical and uterine lymphomas from other entities [3].

The prognosis of the patient depends on the stage of the disease, the location and the subtype lymphoma. The Ann Arbor classification can be made based on findings on the CT scan and bone marrow biopsy [2].

Due to low incidence of primary uterine lymphoma large patient series describing the optimal treatment method are not available. In the scarce available literature it is recommended to treat with hysterectomy and bilateral salpingo-oophorectomy, chemotherapy, irradiation or a combination of these [1].

R-CHOP chemotherapy was shown to improve 5 -year survival rates significantly in patients over 60 years of age with diffuse large B-cell lymphoma [6, 7]. No randomized controlled trials have been performed on the treatment of primary uterine lymphomas, nor are there treatment standards. Therefore it is recommended to individualize the treatment for patients with primary lymphomas of the female genital tract [3].

\section{Conclusion}

A primary uterine lymphoma is a rare malignancy of the female genital tract. Because patients have often nonspecific symptoms, it usually takes longer before the correct diagnosis is made. No evidence-based advice is available for treatment due to its low incidence; therefore, the treatment of patients with a primary uterine lymphoma needs to be individualized.

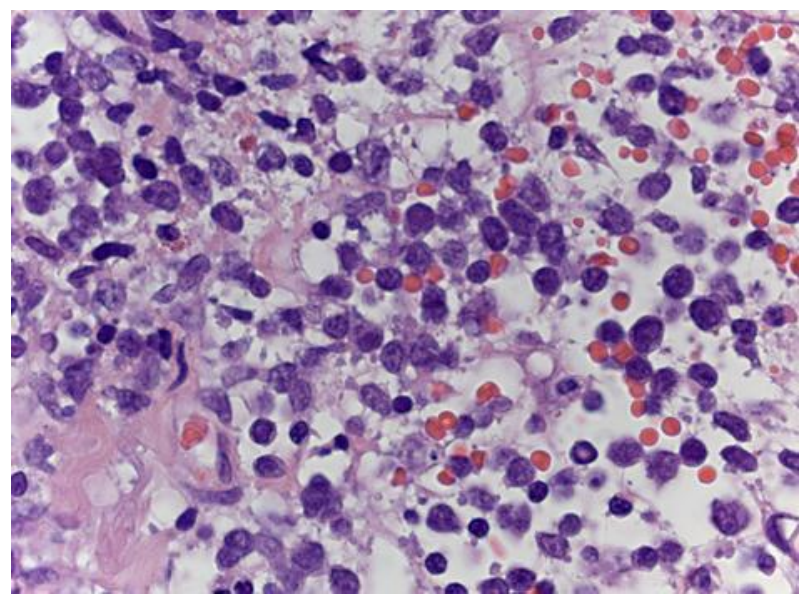

Fig. 1. Histologic specimen showing diffuse large B-cell lymphoma of the uterus (HE staining). 


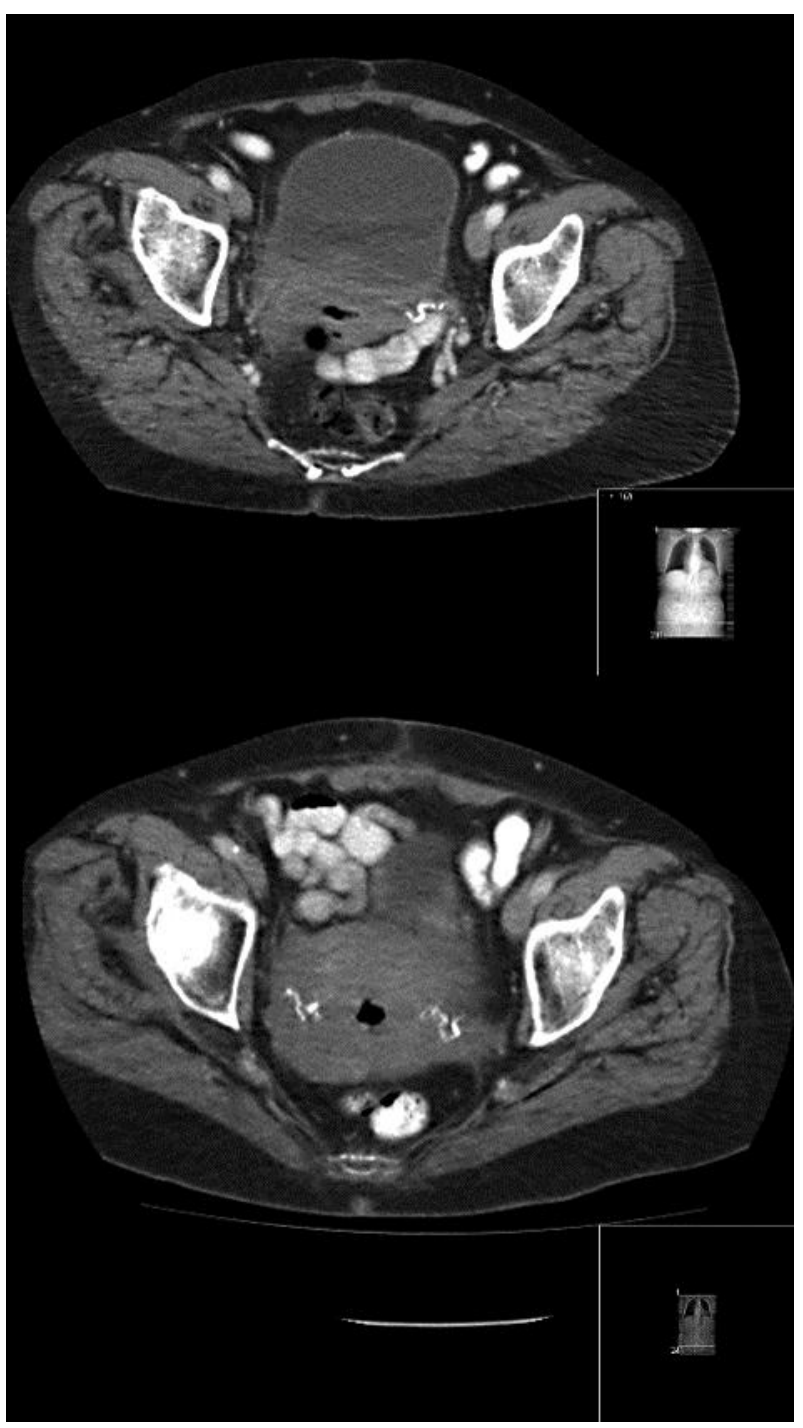

Fig. 2. Above: CT scan showing the primary pelvic mass. Below: CT scan after 6 courses of R-CHOP chemotherapy. Regression of the primary tumour is seen.

\section{References}

1 Shen CJ, Tsai EM, Tsai KB, et al: Primary T-cell lymphoma of the uterine corpus. Kaohsiung J Med Sci March 2007;23:138-140.

-2 Heeren JHM, Croonen AM, Pijnenborg JMA: Primary extranodal marginal zone B-cell lymphoma of the female genital tract: a case report and literature review. Int J Gyn Path 2008;27:243-246.

-3 Frey NV, Svoboda J, Andreadis C, et al: Primary lymphomas of the cervix and uterus: the University of Pennsylvania's experience and a review of the literature. Leuk Lymphoma 2006;47:1894-1901.

4 Fox H, More JR: Primary malignant lymphoma of the uterus. J Clin Pathol 1965;18:723-728.

5 Yamamoto T, Morita K, Iriyama N, et al: Intravascular large B-cell lymphoma of the uterus: a case with favorable clinical outcome. Int J Surg Pathol 2011;19:672-676.

6 Feugier P, Van Hoof A, Sebban C, et al: Long-term results of the R-CHOP study in the treatment of elderly patients with diffuse large B-cell lymphoma: a study by the Groupe d'Etude des Lymphomes de l'Adulte. J Clin Oncol 2005;23:4117-4126.

7 Niitsu N: Current treatment strategy of diffuse large B-cell lymphomas. Int J Hematol 2010;92:231-237. 